\title{
Analysis of the Impact of Interaction Design in Digital Media on User Experience
}

\author{
Jing Zhang \\ School of Media And Art Design,Wuhan Donghu University, Wuhan, China \\ 346591653@163.com
}

KEYWORDS: Digital Media; Interaction Design; User Experience

\begin{abstract}
With the rapid development of information technology characterized computer and network, digital media products have become the main stream of the times, interaction technology gets ubiquitous in people's life. How to create better user experience in the use of digital media products is a hot issue. Here we propose the user experience level of interaction design for digital media, and then through case studies and research to verify the effect of emotional interaction design on the user experience.
\end{abstract}

\section{Introduction}

Nowadays, Digital media technology has brought great convenience to people's life. All digital product are designed to server people, thus interaction design came into being. Specifically, interaction design is the issue about how to create better user experience, and its well-known basic concepts is "design for human", which is exactly terminal services of digital technology products.

However, in interaction design area, most of user experience is purely subjective point of view, and they certainly are not convinced, because different users determine the differences. In this respect, interaction design begins to be an important part in product differentiation strategy.

In the face of digital media and electronic information era today, if designers want to make users accept their interaction products, they should consider developing more humane products.

\section{Interaction Design and Emotional Experience}

Based on the concept of emotional design, we combine the traditional concept of emotion and the interaction design in digital media products, to propose the user experience level of interaction design for digital media, and then through case studies and research to verify the effect of emotional interaction design on the user experience.

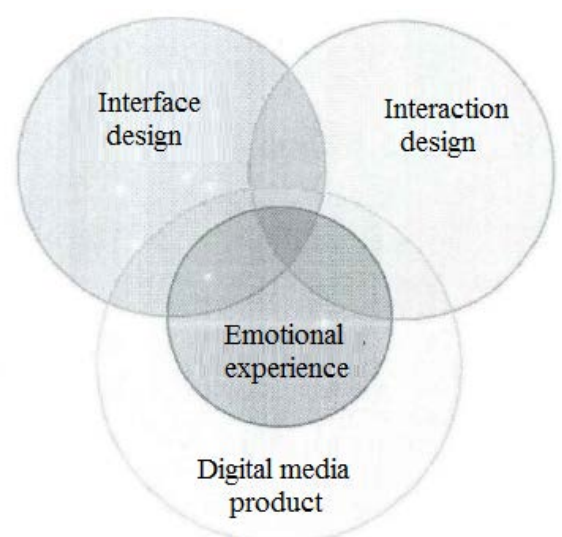

Figure 1. Interaction design and emotional experience 
When the interaction designer is developing a digital media product, his main responsibility is to meet the needs of users through the product. In addition to this, interaction designer should also focus on the emotional aspects of the product from the beginning, and show them in the form of interactive design. Whether the whole website tone and logical structure, or he details of an error prompt, they are all parts of website personality. These emotional factors will be able to determine the customer loyalty of the product to a great extent.

Emotional interaction design can play an important role in the whole process of digital media products design. Interactive design as a technology to help users to be more convenient and enjoyable to use applications, at the beginning stage of product design, it requires visual designers, developers and product managers to participate in it. The cases that applying interaction design and emotional design theory in digital media area is common, and such interaction process to arouse sympathy and deep experience by satisfying users' deep needs is consistent with the concept of internet marketing.

\section{Emotional Experience Level based on Digital Media Interaction Design}

\section{A. The First Level - Emotional Experience of Interface Interaction}

Interface is the window of digital media in contact with the user, which contains the aesthetic function of the shape and the cultural attribute. Before interface interaction design, people need to consider the user's psychological feeling while watching the interface, and meanwhile guess in what way can arouse their emotional needs.

Nearly $90 \%$ of the application will be deleted after the users' initial use, so the first time user open the application for each interface are important. A lot of applications are without any content when login, and this is also a good time to show the application interface design. Take Dropbox for example, the cozy villain icon in Empty state interface would guide users to start creating their valuable collections of information. Such interaction design uses the same style of interface elements with its WEB page, reflecting the continuity and consistency of the product characteristics.

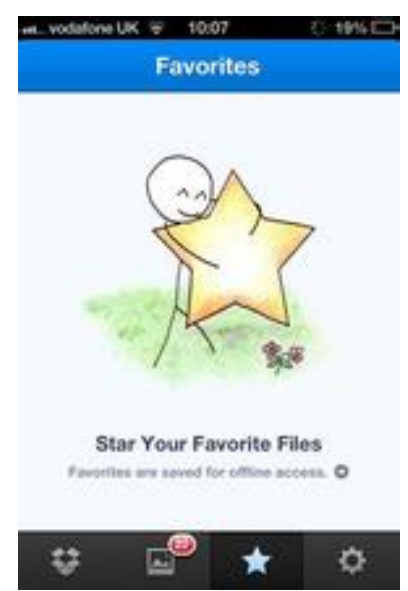

Figure 2. Blank interface design of Drobox

\section{B. The Second Level - Emotional Experience of Operation Interaction}

The operation behavior Meeting human nature will emotionally impress the user. E.g. the multi-touch and page zoom operation firstly used in iPhone is the case that combines intuitive human instinct with advanced technology. Such operation interaction gives the user a pleasurable sensation, and create a new operating model has spread value. 
Of course, people expect digital media can also have a similar emotion feature capability as human, including language, character, expression, tone and action. With the advent of digital media era, this emotional expectation is naturally given interaction design, and Emotional interaction become the main trend of human-computer interaction in the advanced information age.

(1) Facial emotion simulation

Face interaction is the most direct form of communication among people. Expression recognition process substantially is to identify the deformation characteristics and muscle movement facial features to perceive emotions and intentions, and then generate the corresponding expression communicate with users through the database. This intelligent technology is already widely used in digital media products.

Intel has applied its face simulation technology in intelligent mobile phone. It can collect the user's real picture by camera, and abstract it into a simple face line map by certain algorithm. This dynamic facial expression map will change with the change of user expression, and simultaneously show the user's mood. This technique is suitable for dating scene. At the same time it increases interactive quality and fun, but also can guarantee the user's privacy.

(2) Emotional speech analysis

The expression tone of speaker will affect the audience's psychological feeling, so speech emotion interaction is very important for human emotion recognition. In our daily life, speech technology has been used widely, but emotional speech is more complex than simple speech recognition.

With the implantation of Siri into Apple products, virtual dialogue technology has entered people's daily life, but the emotional factors have not been considered yet. User's tone of voice and emotional expression is directly related to the individual's emotional experience and requirement. Virtual dialogue technology in digital media should be able to identify the user's emotion, thus it can help provide corresponding service. The product Emotional Phone Book could classify the contacts in the address book by analyzing the emotional elements in speech communication.

(3) Anthropomorphic elements

It is brilliant to simulate human voice, eyes, facial expressions and other details by digital technology. Currently, some applications realize these techniques by establishing simulation character. These simulation characters must have appearance to make people feel comfortable, and simulate human behavior and action of real world by computer. E.g. the application ShuangShuang, could not only receive user emotional physiological signals, but also can make intelligent response to user through mobile device.

(4) Physiological emotion detection

Taking advantage of the built-in functions of mobile media devices, people have continually developed some physiological detection applications. These applications can feel the heartbeat and other indicators to judge the user's mood by means of capillary. The physiological and emotional performance is extremely objective, and sometimes it can examine thoroughly user's emotional state that himself fail to be aware of. E.g. application Heart Fitness can sense the beating of capillaries to detect heart rate through the mobile device flashlight function, and then generate reports, to tell user about his health state and indicators comparison. This objective data provides comprehensive reference index to determine user's emotion. 


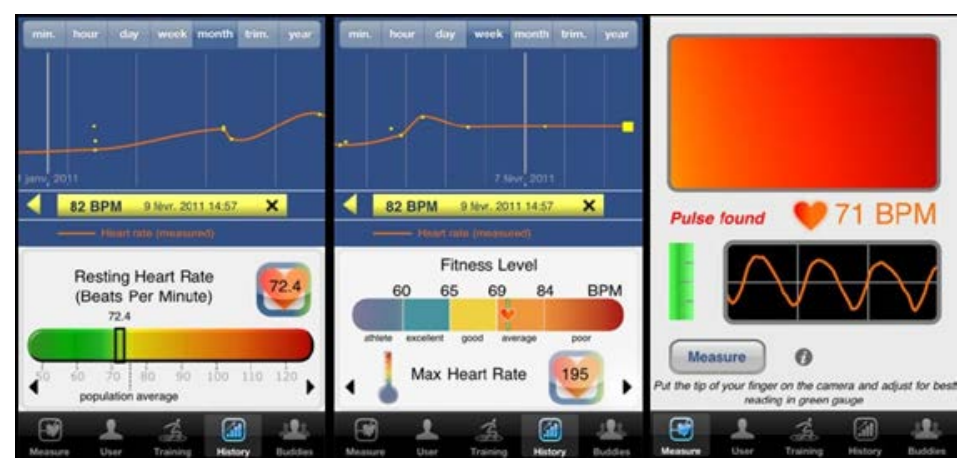

Figure 3. Heart Fitness

\section{The Second Level - Emotional Experience of Product Nature}

Nature layer of products need to mobilize the user's inner feelings. Product of good emotional experience can give users a surprise, that is, to meet the basic needs of consumers and give more added emotional value. E.g. Douban Radio would automatically recommend user's favorite music according to his listening habits. Such emotional interaction function will cultivate users' sense of dependence and loyalty.

\section{References}

[1] Mendes M S, Carvalho A L, Furtado E, et al. Towards for Analyzing Alternatives of Interaction Design Based on Verbal Decision Analysis of User Experience[J]. International Journal of Interactive Mobile Technologies, 2010, 4(2).

[2] Lau N, Zhang M. Interaction Design and the Construction of User Experience in Portable Learning Utility Solutions[J]. Design Principles \& Practice An International Journal, 2011(4):453-466.

[3] Wurhofer D, Fuchsberger V, Meneweger T, et al. Insights from User Experience Research in the Factory: What to Consider in Interaction Design[M]// Human Work Interaction Design. Work Analysis and Interaction Design Methods for Pervasive and Smart Workplaces. Springer International Publishing, 2015.

[4] Redlarski K, Garnik I, Sikorski M. User Experience research in the user-system interaction design[J]. Business Informatics, 2011(22):13.

[5] Yuan J, Zhang W, Xing R. User experience interaction design for digital educational games[C]// Society of Photo-Optical Instrumentation Engineers (SPIE) Conference Series. Society of Photo-Optical Instrumentation Engineers (SPIE) Conference Series, 2014:4177-4180. 